\title{
Effect of counselling during pulmonary rehabilitation on self-determined motivation to be physically active for people with chronic obstructive pulmonary disease: a pragmatic RCT
}

Anne-Kathrin Rausch Osthoff ${ }^{*}$, Swantje Beyer ${ }^{3}$, David Gisi ${ }^{2}$, Spencer Rezek ${ }^{2}$, Ariane Schwank ${ }^{2}$, André Meichtry ${ }^{1}$, Noriane A. Sievi ${ }^{4}$, Thomas Hess $^{3+}$ and Markus Wirz ${ }^{1+}$

\begin{abstract}
Background: Counselling is considered to be a promising approach to increasing physical activity (PA) in people with chronic obstructive pulmonary disease (COPD). The aim of the current study was to investigate whether a PA counselling program for people with COPD, when embedded in a comprehensive outpatient pulmonary rehabilitation (PR) program, increased their daily PA.
\end{abstract}

Methods: A two-armed, single blind randomized controlled trial was conducted as a component of a 12-week outpatient pulmonary rehabilitation program. The participants randomized into the intervention group received five counselling sessions, based on the principles of motivational interviewing (MI), with a physiotherapist. The participants'steps per day and other proxies of PA were measured using an accelerometer (SenseWear Pro ${ }^{\circledR}$ ) at baseline, at the end of the PR program, and three months later. The group-by-time interaction effect was analyzed.

Results: Of the 43 participants, 17 were allocated to the intervention group and 26 to the usual-care control group (mean age 67.9 $\pm 7.9 ; 21$ (49\%) males; mean FEV1 predicted $47.1 \pm 18.6)$. No difference between groups was found for any measure of PA at any point in time.

Conclusions: In this study, counselling, based on Ml, when embedded in a comprehensive PR program for people with COPD, showed no short-term or long-term effects on PA behavior. To investigate this potentially effective counselling intervention and to analyze the best method, timing and tailoring of an intervention embedded in a comprehensive outpatient PR program, further adequately powered research is needed.

Trial registration: Clinical Trials.gov NCT02455206 (05/21/2015), Swiss National Trails Portal SNCTP000001426 (05/21/2015).

Keywords: Behavioral change, Physical activity promotion, COPD, Motivational interviewing

*Correspondence: anne-kathrin.rauschosthoff@zhaw.ch

†Thomas Hess and Markus Wirz shared load

${ }^{1}$ Institute of Physiotherapy, School of Health Professions, ZHAW Zurich University of Applied Sciences, Katharina-Sulzer-Platz 9, 8400 Winterthur, Switzerland

Full list of author information is available at the end of the article

\section{Background}

Physical activity (PA) promotion for patients with Chronic Obstructive Pulmonary Disease (COPD) is a research field of great importance. Both the American Thoracic Society (ATS) and the European Respiratory Society (ERS) have stressed that long-term self-management and adherence to exercise at home should be the 
primary goal of pulmonary rehabilitation programs (PR) [1]. Sound evidence has shown that physical inactivity in COPD patients is associated with an increased number of hospitalizations [2-4], and that it is the strongest predictor of all-cause mortality $[3,5]$. Dyspnea, induced by physical exercise, may lead to a shift in a patient's lifestyle, resulting in a vicious cycle of decreased exercise tolerance. This, in turn, further reduces PA levels and increases the likelihood of social isolation and depression [6]. Conversely, the benefits of adequately dosed PA have been sufficiently proven [7-9]. PR, enhancement of exercise capacity, and promotion of an active life style are the cornerstones of non-pharmacological COPD management $[10,11]$. However, poor adherence is common and changing behavior through enhancing motivation is a daily task for therapists [12-14]. Pitta \& Burtin emphasize the importance of having PA coaches as members of the PR team, for the purpose of, helping patients set and progress PA goals, reinforcing COPD self-care skills, supporting efforts to monitor their activities and symptoms, assisting with problems, solving PA barriers, and troubleshooting device or technology issues [15]. The use of Motivational Interviewing (MI) techniques for PA counselling have been supported within the PR community $[10,16]$.

Miller and Rollnik ([17], p12) describe MI as a "collaborative conversation style for strengthening a person's own motivation and commitment to change". It focuses on the exploration and evocation of a person's intrinsic motivation to change their perception towards a specific goal, e.g., daily PA. Hereby, MI emphasizes the ambivalent attitude of a person to behavioral change, rather than seeing a person as unmotivated. Consequently, it is the therapist's intention to detect a patient's ambivalence and to try to resolve it. This attitude is called "MI spirit" and is based on the four principles of: collaboration, compassion, evocation and acceptance [17]. The process to change the behavior associated with PA can be divided into two phases; firstly, creation of the motivation to change, and secondly, the commitment to change. A non-judgmental and empathic communication style can help patients to make various behavior changes, e.g. quit smoking $[18,19]$, or to enhance treatment adherence [20].

In 2016, Lahham and colleagues showed that studies adding PA counselling, based on MI, to PR [21-24] resulted in an effect exceeding the established minimal important difference of 599 steps per day [25]. However, these pooled effects were demonstrated in the short-term at three months, but were not maintained in the longerterm at six to 15 months [25].

Since 2016, several other studies have investigated the effect on PA behavior of adding MI-based PA counselling to PR, measured by steps per day. Benzo and colleagues (2018) did not find any difference in PA between groups when investigating the effect of MI-based health coaching compared to usual care (PR) in people with COPD after hospitalization due to exacerbation [26]. A similar result was found by Arbilliga-Etxarri and colleagues (2018) investigating the effect of a 12-week program of MI-based PA promotion counselling, which was combined with urban walking training, compared to usual care. However, a sub-analysis, including only those patients who were willing and adherent, showed a significant effect on the number of steps per day [27].

While these studies indicate some positive effects of MI-counselling on PA in the short-term, no conclusive evidence is available on which strategy, using MI techniques in the PR setting, could be effective in promoting long-term adherence to an active lifestyle. Both patients and health professionals have emphasized that the coaching style and the relationship are important aspects of PA counselling [28]. Furthermore, the individual stage of change, based on the transtheoretical model of health behavior change [29], may be relevant to tailoring MIcounselling. Data from well-designed interventional studies for people with COPD that look at the willingness to actually change habitual behavior are urgently needed [30].

The aim of the current study was to investigate whether a PA counselling program for people with COPD, when embedded in a comprehensive outpatient pulmonary rehabilitation (PR) program, increased their daily PA.

\section{Methods \\ Design and ethics}

The study design was a pragmatic, prospective two-arm single-blinded randomized controlled trial comparing a PA counselling group (IG) with a control group (CG) of usual-care. The regional ethics committee (EC,Canton Zurich) approved the study on $4^{\text {th }}$ May 2015 (PB_201601,523). Data collection was conducted in accordance with Good Clinical Practice protocols and the Declaration of Helsinki principles. The COPD participants received written and oral information about the study. Written informed consent was obtained from all participants prior to baseline measurement. The study was registered on the website of http://www.ClincalTrails.gov with the identifier NCT02455206 (27/05/2015), as well as on the Swiss National Trails Portal SNCTP000001426 (05/21/2015). The study protocol was also published [31].

\section{Setting}

This study was conducted at the Cantonal Hospital Winterthur (KSW), Institute of Physiotherapy and Division of Pneumology, Switzerland. 


\section{Participants}

Participants fulfilling the following inclusion criteria were eligible for the study: informed consent, as documented by signature; age 40-90 years and confirmed COPD (GOLD grades 2-4, according to GOLD-guidelines) [6]; German-speaking; and, planned participation in PR program. Exclusion criteria were applied as previously described [31]: summarized as, mental or physical disability, severe uncontrolled co-morbidities. Any adverse events occurring during the outpatient PR, such as injuries, increased respiratory symptoms, or cardiac events, were recorded and reported to the EC.

\section{Recruitment and randomization}

All individuals with confirmed COPD and referral to the pulmonary division of the KSW for PR were invited consecutively to participate in the study. No public advertisement was made. Randomization was performed by an independent person, based on a list generated by computer software ( $\mathrm{R}$ statistical software). Additionally, stratification was used to ensure balanced exercise capacity (6-min walking test, 6MWD) in both groups at baseline, which could influence how sustainable someone can implement regular PA in everyday life. The randomization was accessible only to the responsible members of the research team. The assessors and statistician were blinded but blinding of participants and therapists was not feasible.

\section{Intervention}

\section{Pulmonary rehabilitation}

A comprehensive PR was performed [32], according to the previously published study protocol [31], based on education and tailored individual intervention. Participants attended a PR program at the outpatient clinic twice weekly (each session $1.5 \mathrm{~h}$ ) and, additionally, performed a supervised Nordic Walking training outdoors once a week (1.5-h session). Thus, a total of 36 physical training sessions were performed during the 12-week PR.

The sessions included dynamic strength training (performed on conventional strength training equipment in a seated position) and cardio-pulmonary endurance training. For the strength training, patients started at $70 \%$ of their initial one-repetition maximum (1RM) and completed 2 cycles of 6-12 repetitions of isotonic muscle contractions [33-35], with a resting period of 1-2 min between the series. If participants felt able to perform three sets of more than 15 repetitions without any difficulty, the training load was increased stepwise by $5 \%$ of the 1 RM. Cardio-pulmonary endurance training was performed either on a cycle ergometer (sitting or recumbent) or a treadmill [33-35]. The initial training intensity was set at $70-80 \%$ of maximal exercise effort for $25 \mathrm{~min}$. Increases in workload were based on symptom scores, which were assessed with a Borg scale. If patients were not able to perform $25 \mathrm{~min}$ of endurance training, interval endurance training was performed [36].

\section{Intervention group}

Participants allocated to the IG received the usual 12-week PR program plus five face-to-face at weeks $1,3,6,9$ and 12, 30-min counselling sessions, performed according to the principles of MI. The PA counselling was provided by two physiotherapists (MSc level), who were not involved in the usual PR program. They were trained prior to the study by an experienced MI-trainer and member of the MINT (Motivational Interviewing Network of Trainers).

\section{Evaluation of counsellor communication skills}

Counselling sessions were audiotaped. A fidelity check evaluated the general session content, as well as random 20-min segments of the conversations, which were assessed by an external, independent MI-expert, according to the Motivational Interviewing Treatment Integrity (MITI 4.2.1) [37, 38] criteria. Feedback was provided to the counsellors to strengthen their skills and consistency with the study intervention [39].

\section{Measurements}

Measurements, described in detail elsewhere [31], were performed at baseline (T0), after 12 weeks PR (T1), and after 24 weeks (follow-up, T2). Outcomes and Assessments are described in Table 1.

\section{Analysis}

The mean and standard deviation (SD) were presented for demographic and disease-specific data, median and associated range for continuous data, and frequencies (percentages) for categorical variables.

The threshold for valid data from the accelerometer was set at four days, with a minimum wearing time of $22.5 \mathrm{~h} /$ day [49]. The choice of days was decided as follows to cover a wide range of daily routine and associated activity levels [50]: (a) one day on the weekend, preferably Sunday; (b) the days during the week with the highest, the second highest, and the lowest time of activity.

For each continuous outcome, the following Linear Mixed Model was fitted to the data. The model for observation $Y_{i j k}$ was

$$
\begin{aligned}
Y_{i j k} & =\mu_{i j}+U_{i k}+\varepsilon_{i j k}, i=1,2 ; \\
j & =1, \ldots, 3 ; k=1, \ldots, n_{i}[51],
\end{aligned}
$$

with $\mu_{i j}$ as the mean for group $i$ and time $j, U_{i k}$ as the random effect of subject $k$ in group $i$ and $\varepsilon_{i j k}$ as measurement 
Table 1 Outcomes and assessments measured

\begin{tabular}{|c|c|c|}
\hline \multicolumn{2}{|l|}{ Outcome } & \multirow{2}{*}{$\begin{array}{l}\text { Assessment } \\
\text { Accelerometer (SenseWear Pro }{ }^{\circledR} \text { armband; BodyMedia, Inc., Pittsburgh, PA, USA) [40] }\end{array}$} \\
\hline Primary & PA: Mean number of steps per day & \\
\hline Secondary & $\begin{array}{l}\text { Other proxies of PA } \\
\text { metabolic equivalent (MET), } \\
\text { total energy expenditure (TEE), } \\
\text { physical activity level (PAL) }\end{array}$ & Accelerometer (SenseWear Pro ${ }^{\circledR}$ armband; BodyMedia, Inc., Pittsburgh, PA, USA) [40] \\
\hline \multirow[t]{7}{*}{ Tertiary: } & Awareness of PA behavior & International Physical Activity Questionnaire (IPAQ) [41, 42] \\
\hline & Individual motivation towards exercising & Behavior Exercise Regulation Questionnaire (BREQ-3) $[43,44]$ \\
\hline & $\begin{array}{l}\text { The patient's willingness to change their } \\
\text { habitual behavior }\end{array}$ & Physical Activity Stage of Change Assessment Tool (PASC) [45] \\
\hline & Health-related quality of life & Chronic Respiratory Disease Questionnaire (CRQ) $[46,47]$ \\
\hline & Impact of COPD on health status & COPD Assessment Test (CAT) [48] \\
\hline & PR adherence & Number of sessions participation \\
\hline & Exercise capacity & $\begin{array}{l}\text { Six-minute walking test (6MWT), } \\
\text { One-minute Sit to stand (1STS), Pulmonary function }\end{array}$ \\
\hline
\end{tabular}

PA, physical activity; PR, pulmonary rehabilitation; COPD, chronic obstructive pulmonary disease

error. Normal distributions were assumed for the random intercepts and the errors.

The parameters of interest were the between-group differences in change between the time points $1-2,1-3$ and $2-3$, i.e., the group-by-time interactions. A global test for the group-by-time interaction effect was performed and 95\% confidence intervals for the interaction effects were computed by adjusting for multiple testing, where necessary. Residual analysis was performed to check the model assumptions.

All analyses were performed using the $\mathrm{R}$ statistical software R version 4.0.3 (2020-10-10) [51, 52].

\section{Results}

Patient recruitment and enrolment were started in June 2015 and ended in March 2020 (ahead of schedule due to the Covid-19 pandemic). The patient flow is presented in the Consolidated Standards of Reporting Trial diagram (Fig. 1).

Of the 135 patients screened, 43 (32\%) were included and allocated, either to the IG $(n=17)$ or to the CG $(n=26)$. One person allocated to CG dropped out before starting PR. At baseline, both groups were balanced in terms of gender, age, PA-related primary and secondary outcomes (Table 2). Patients had a median exercise session attendance rate of 36 days (range 6-36; mean 33.3, SD 7.0).

Table 3 shows the outcomes for both groups at the three measurement time points. Neither the primary outcome of 'steps per day', nor the other proxies of PA (mean MET, TEE and PAL), showed any group-by-time differences (Table 4 and Fig. 2). Table 5, describing the PA stage of change for both groups, indicates that the majority of participants are in the stages of preparation, action or maintenance at all time points of measurement. The intervention fidelity of the counsellors was rated as sufficient.

\section{Discussion}

This study showed that counselling for people with COPD, based on MI principles, did not lead to an increase in daily PA, either during or after participation in a comprehensive outpatient PR. Despite some tendencies, there was no robust group-by-time difference for a proxy of PA, or any other outcome.

This pragmatic study is deemed to be underpowered. The change in 'steps per day' corresponds to the minimal important difference (MID) [53], but is not statistically significant. The inhomogeneity of study participants is clear, thus limiting the validity of the findings. However, the individual PA behavior of the responders and nonresponders towards counselling can be identified. Some individuals benefited from counselling and responded well to the intervention. A qualitative analysis revealed four types of COPD patients, based on their perceived PA level, quality of motivation, and coping strategies. This might be useful to better understand the responders to counselling interventions [54].

These findings conform to the current literature. Although MI-counselling appears to be a promising intervention to promote behavioral change in people with COPD [55], current results in the context of PA promotion are inconclusive. Two studies [23, 24], measured by steps per day and based on MI-techniques and pedometer feedback, showed positive short and long-term effects of counselling. However, another two studies [21, 22] showed no additional effect of MI-counselling on PA levels. A meta-analysis of these 


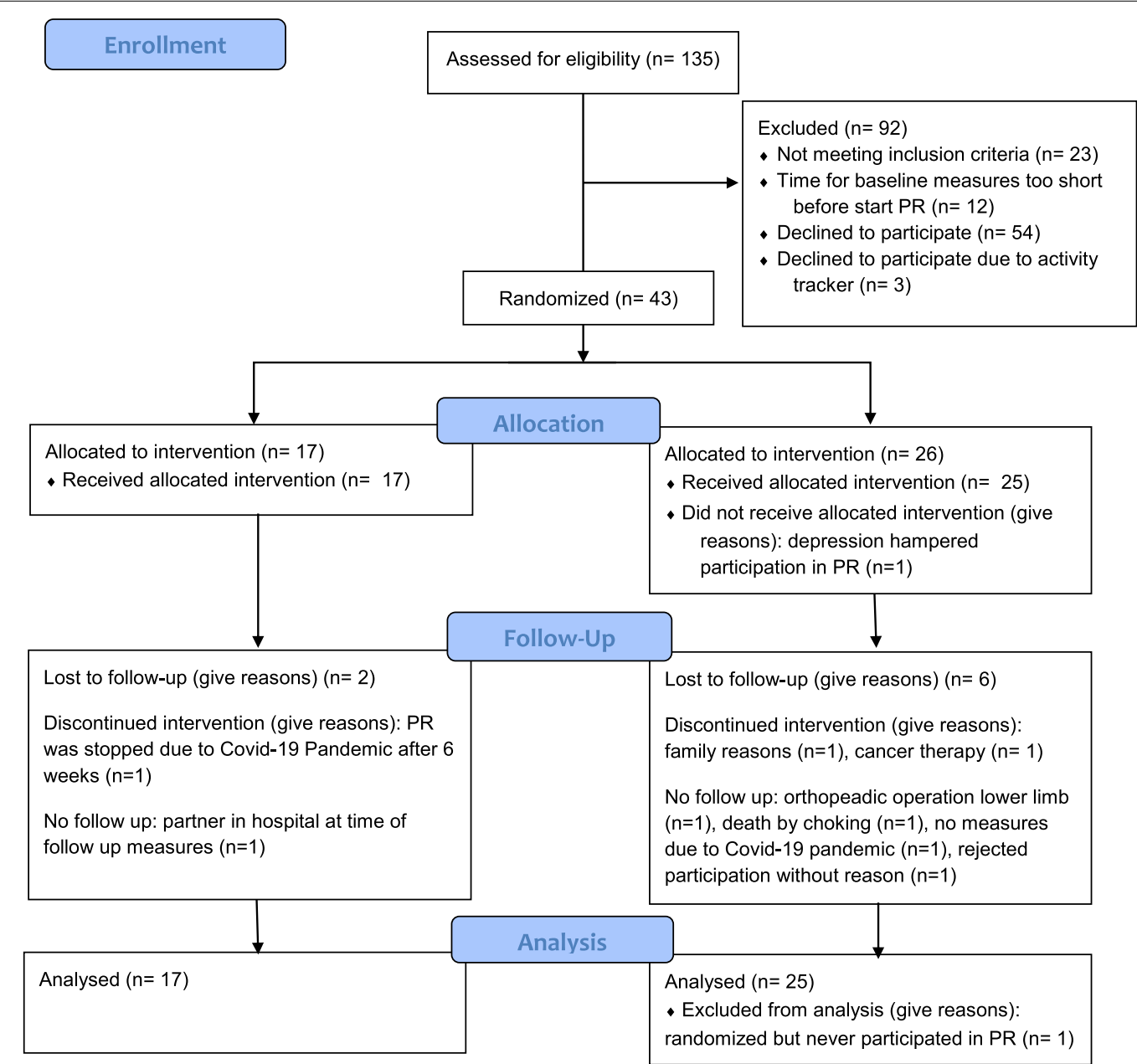

Fig. 1 Consort diagram describing the flow of patients. PR, pulmonary rehabilitation; IG, intervention group receiving physical activity counselling, $\mathrm{CG}$, control group receiving usual care

four studies showed small to moderate effects at three months that tended towards statistical significance when PA counselling was added to PR (compared to PR only), but showed no long-term effects [25]. Additionally, Benzo and colleagues [26] concluded that telephone-based MI, following PR, lead to reduced hospital readmissions, but that it had no effect on PA related outcomes.

In this study, a pedometer for activity tracking and feedback was not used. An alternative device without a feedback function was utilized. Raising awareness through feedback and self-monitoring are recognized techniques to support behavioral change [56]. Findings from Altenburg [23] and Cruz [24] indicated that a pedometer support had short and long-term effects on the PA level. It could be a disadvantage not to offer direct feedback, such as with a pedometer, to every participant [57]. However, it is possible that the external feedback by pedometer could have raised patients' motivation to be physically active and, consequently, decreased the effect of counselling between groups even more.

Donaire-Gonzales and colleagues [7] evaluated the interaction of quantity and intensity of PA and their effects on COPD hospitalization risk. For people with low average PA intensity, the risk of COPD hospitalization was reduced by $20 \%$ for every additional $1^{\prime} 000$ daily steps. Contrarily, and a little surprisingly, adding steps to an already high-intensity PA did not result in any risk reduction. This leads to the question of whether counselling should focus on the more active patients, i.e., those probably more willing to adopt an adequate PA behavior, or whether it is more important to specifically address inactive patients, who are probably less willing to change and less likely to adopt a sufficient PA behavior. Regardless of ethical considerations, a sophisticated counselling strategy performed by well-trained counsellors is needed. Although the best behavioral change strategies are still under debate, counselling has been recommended [58]. 
Table 2 Characteristics of patients at baseline

\begin{tabular}{lcc}
\hline Patient characteristics at baseline & Control (n=25) & $\begin{array}{c}\text { Intervention } \\
(\mathbf{n}=\mathbf{1 7})\end{array}$ \\
\hline Gender, m (\%) & & $9(53)$ \\
Age in years, mean (SD) & $12(48)$ & $70(7)$ \\
BMI, mean (SD) & $67(9)$ & $27(5)$ \\
Active smoker, yes (\%) & $25(7)$ & $8(47)$ \\
Packyears, mean (SD) & $8(32)$ & $53(22)$ \\
N of cigarettes per day, mean (SD) & $46(22)$ & $7(3)$ \\
Years since stopped smoking, mean (SD) & $15(12)$ & $12(10)$ \\
N of exacerbations in last 12 months, mean (SD) & $9(8)$ & $0.3(0.4)$ \\
6MWD, m, mean (SD) & $0.4(0.7)$ & $470(90)$ \\
FVC, I, mean (SD) & $448(72)$ & $2.7(0.7)$ \\
FVC \%, mean (SD) & $2.6(0.8)$ & $85.5(26)$ \\
FEV1, I, mean (SD) & $77.1(14)$ & $1.3(0.4)$ \\
FEV1, \%, mean (SD) & $1.1(0.4)$ & $52.5(20)$ \\
FEV1_FVC, mean (SD) & $45.6(16)$ & $56.7(14)$ \\
\hline
\end{tabular}

$\mathrm{n}$, number; SD, standard deviation; \%, percentage; BMI, body mass index; 6MWD, six minutes walking distance; m, meter; FEV1, forced expiratory volume in 1 s; FVC, forced vital capacity; I, volume

Important messages for COPD patients are: (a) Latest PA recommendations underpin the beneficial effects of PA for people with chronic diseases; since there is no lower threshold for benefits from PA, some benefits are intermediate [59]; (b) Adults with COPD should do moderate (150-300 $\mathrm{min} /$ week) and/or vigorous (75-150 $\mathrm{min} /$ week) cardiovascular exercise, twice weekly strength exercise, flexibility and neuromotor exercises [59]; (c) The guidelines acknowledge that people with chronic conditions should engage in PA according to their abilities and might need advice on the types and amounts of PA appropriate to their individual needs and abilities [59]; (d) Reduction of sedentary behavior is especially beneficial for people with low PA.

Frequency of exercise training is a key factor to consider, since it is assumed that frequent and structured training is more effective in increasing PA levels than irregular training. Studies involving exercise training of three times per week have demonstrated considerable PA improvement [60]. Furthermore, authors have concluded that longer-lasting PR programs achieve better improvements in PA when compared to short-term PR programs [60]. In fact, studies in which the PR program lasted longer than 12 weeks achieved better PA levels than those in which the training was shorter ( $\leq 12$ weeks). The longitudinal study of Pitta and colleagues stated that exercise training duration is fundamental. In this study, patients trained for 12 weeks and revealed a minor, nonsignificant PA increase. The effect on PA only became significant for participants with supervised training over six months [61]. Since the participants in this current study participated in only 12 weeks of PR and trained 3 times weekly, it might explain the lack of significant difference in PA between the groups.

Wempe and Wijkstra [62] confirmed that people usually needed a minimum of three months to change a habit: in fact, "one needs three months to train the muscle, but six months to train the brain" [63]. To accurately investigate interventions and achieve a successful outcome, frequent supervised exercise training should be provided over a longer period. It is generally acknowledged that the chance of success of a behavioral change program increases when the intervention period exceeds six months. Prochaska [29] mentioned that, according to the Stages of Change model, individuals trying to change their behavior should initiate and remain in an "action" phase for six months prior to moving into the subsequent "maintenance" period. This might explain why the IG in our study only improved in the period T2.

The timing of counselling interventions might also be relevant to its effectiveness. In our study, participants received five 30-min counselling sessions at weeks $1,3,6,9$, and 12 . To facilitate the translation of regular exercise in the PR setting into that of daily life after $\mathrm{PR}$, the counselling might have been more effective as a "bridging intervention" at the very end of, or following, PR. This would take into account the fact that COPD patients are generally less active directly following PR (need to recover), and that their level of PA may increase again later [64]. Whether a combination of presence and remote (digital) counselling could be an effective intervention needs to be evaluated, since evidence on 
Table 3 Primary and secondary outcomes

\begin{tabular}{|c|c|c|c|c|c|c|c|}
\hline Primary and secondary outcomes & Group & $\mathrm{n}$ at T0 & T0 & $\mathrm{n}$ at T1 & T1 & $\mathrm{n}$ at $\mathrm{T2}$ & $\mathrm{T} 2$ \\
\hline \multirow[t]{2}{*}{ Steps per day, mean (SD) } & IG & 14 & $4987(2751)$ & 13 & $5026(2859)$ & 12 & $6054(3560)$ \\
\hline & CG & 18 & $5581(3413)$ & 16 & $5651(3582)$ & 14 & $5180(2803)$ \\
\hline \multirow[t]{2}{*}{ TEE per day, mean (SD) } & IG & 14 & $2324(746)$ & 13 & $2196(833)$ & 12 & $2587(1098)$ \\
\hline & CG & 18 & $2440(472)$ & 16 & $2208(416)$ & 14 & $2163(485)$ \\
\hline \multirow[t]{2}{*}{ MET per day, mean (SD) } & IG & 14 & $1.3(0.3)$ & 13 & $1.3(0.3)$ & 12 & $1.3(0.2)$ \\
\hline & CG & 18 & $1.5(0.5)$ & 16 & $1.4(0.2)$ & 14 & $1.3(0.3)$ \\
\hline \multirow[t]{2}{*}{ PAL per day, mean (SD) } & IG & 14 & $1.6(0.3)$ & 13 & $1.5(0.3)$ & 12 & $1.5(0.3)$ \\
\hline & CG & 18 & $1.6(0.3)$ & 16 & $1.5(0.1)$ & 14 & $1.5(0.2)$ \\
\hline \multirow[t]{2}{*}{ Time with low MET, minutes per day, mean (SD) } & IG & 14 & $1321(130)$ & 13 & $1275(183)$ & 12 & $1277(144)$ \\
\hline & CG & 18 & $1299(137)$ & 16 & $1329(75)$ & 14 & $1298(156)$ \\
\hline \multirow[t]{2}{*}{ Time with medium MET, minutes per day, mean (SD) } & IG & 14 & $64(83)$ & 13 & $53(44)$ & 12 & $57(61)$ \\
\hline & CG & 18 & $78(62)$ & 16 & $57(48)$ & 14 & $61(39)$ \\
\hline \multirow[t]{2}{*}{ Time with high MET, minutes per day, mean (SD) } & IG & 14 & $2(4)$ & 13 & $1(3)$ & 12 & $3(5)$ \\
\hline & CG & 18 & $19(33)$ & 16 & $3(6)$ & 14 & $4(10)$ \\
\hline \multirow[t]{2}{*}{ Time with very high MET, minutes per day, mean (SD) } & IG & 14 & $0.2(1.1)$ & 13 & $0(0)$ & 12 & $1(3)$ \\
\hline & CG & 18 & $1.7(3.7)$ & 16 & $0.1(0.3)$ & 14 & $0.9(3.2)$ \\
\hline \multirow[t]{2}{*}{ Average sitting time per day, minutes, mean (SD) } & IG & 13 & $2155(1276)$ & 13 & $1536(827)$ & 12 & $1888(792)$ \\
\hline & CG & 18 & $2154(960)$ & 16 & 1918(911) & 14 & 1709 (879) \\
\hline \multirow[t]{2}{*}{ IPAQ total METs of PA a week, mean (SD) } & IG & 13 & $4246(4715)$ & 12 & $8819(9632)$ & 12 & $5891(5363)$ \\
\hline & CG & 18 & $2437(2731)$ & 13 & $6400(5288)$ & 14 & $5291(5798)$ \\
\hline \multirow[t]{2}{*}{ IPAQ, mean minutes sitting time per day, mean (SD) } & IG & 14 & $2155(1276)$ & 12 & $1536(826)$ & 12 & $1887(791)$ \\
\hline & CG & 18 & $2154(969)$ & 13 & $1918(911)$ & 14 & $1708(878)$ \\
\hline \multirow[t]{2}{*}{ CRQ total, mean (SD) } & IG & 17 & $17(4)$ & 15 & $21(4)$ & 14 & $21(3)$ \\
\hline & CG & 25 & $19(5)$ & 21 & $21(5)$ & 16 & $19(4)$ \\
\hline \multirow[t]{2}{*}{ CAT, mean (SD) } & IG & 13 & $21(8)$ & 9 & $17(6)$ & 0 & NA \\
\hline & CG & 21 & $15(7)$ & 19 & $12(6)$ & 0 & NA \\
\hline \multirow[t]{2}{*}{ 1-Sit-to-stand, mean (SD) } & IG & 17 & $24(7)$ & 15 & $30(9)$ & 15 & $26(8)$ \\
\hline & CG & 24 & $24(9)$ & 21 & $30(11)$ & 18 & $27(9)$ \\
\hline \multirow[t]{2}{*}{ 6-min walking distance, m, mean (SD) } & IG & 17 & $469(90)$ & 16 & $497(89)$ & 15 & $477(93)$ \\
\hline & CG & 25 & $447(71)$ & 23 & $469(98)$ & 18 & $469(77)$ \\
\hline \multirow[t]{2}{*}{ BREQ3, score, mean (SD) } & IG & 14 & $13(7)$ & 12 & $16(4)$ & 12 & $14(6)$ \\
\hline & CG & 16 & $7(10)$ & 18 & $16(5)$ & 14 & $13(6)$ \\
\hline
\end{tabular}

IG, intervention group receiving physical activity counselling; $C G$, control group receiving usual care; $S D$, standard deviation; TEE, total energy expenditure; $M E T$, metabolic equivalent of task; PAL, physical activity level; IPAQ, International Physical Activity Questionnaire; CRQ, Chronic Respiratory Disease Questionnaire; CAT, COPD Assessment Test; min, minutes; BREQ, Behavioral Regulation in Exercise Questionnaire

telerehabilitation in patients with COPD is growing fast [65-69]. However, research information on tele-counselling in a PR context is scarce [70]. Finally, whether counselling is needed by all patients, or whether it is more effective in people willing to engage in the additional counselling service, needs to be investigated.

Even though adherence was good in this study, poor adherence in the general context of PR is common [71]. Lifestyle changes, which are part of the PR goals [32], require a great degree of effort from both clinicians and patients. Perceived beneficial effects and positive personal perceptions of MI encourage clinicians to learn and use MI in their daily working routines. Patients participating in this study appreciated the counselling intervention [54]. However, the poor recruitment rate of $32 \%$ suggests that patients did not initially find a study to evaluate a counselling intervention attractive. Possible reasons for this might be that the explanation of the counselling was not understood, or that patients did not see the need for self-induced behavioral change. Counselling, based on MI techniques, is already implemented in many places. A number of stakeholders, such as the Swiss Lung Foundation [72] and the Working Group Pulmonary Rehabilitation of the three Germanspeaking countries $(\mathrm{CH}, \mathrm{GER}, \mathrm{AT})$ [73], promote the 
Table 4 Group-time interactions for the periods T1-T0, T2-T0 and T2-T1

\begin{tabular}{|c|c|c|c|c|c|c|c|c|}
\hline Outcome & Time Period & Estimate & SE & df & Lower $\mathrm{Cl}$ & Upper Cl & t-value & $p$-value \\
\hline \multirow[t]{3}{*}{ Mean steps } & T1-T0 & 323 & 773 & 49.1 & -1588 & 2235 & 0.42 & 0.96 \\
\hline & T2-T0 & 888 & 795 & 48.9 & -1078 & 2853 & 1.12 & 0.61 \\
\hline & $\mathrm{T} 2-\mathrm{T} 1$ & 564 & 803 & 48.3 & -1421 & 2550 & 0.70 & 0.83 \\
\hline \multirow[t]{3}{*}{ Mean MET } & T1-Т0 & 0.13 & 0.13 & 51.0 & -0.13 & 0.40 & 0.25 & 0.51 \\
\hline & T2-T0 & 0.13 & 0.13 & 50.7 & -0.14 & 0.40 & 1.15 & 0.58 \\
\hline & $\mathrm{T} 2-\mathrm{T} 1$ & -0.00 & -0.00 & 49.8 & -0.28 & 0.27 & -0.06 & 0.99 \\
\hline \multirow[t]{3}{*}{ Mean time low MET } & T1-T0 & -77.5 & 66.8 & 55.2 & -242 & 87.1 & -1.16 & 0.58 \\
\hline & T2-T0 & -45.7 & 66.8 & 55.2 & -212 & 123.8 & -0.66 & 0.88 \\
\hline & $\mathrm{T} 2-\mathrm{T} 1$ & 31.8 & 70.2 & 54.5 & -141 & 204.8 & 0.45 & 0.95 \\
\hline \multirow[t]{3}{*}{ Mean time moderate MET } & T1-T0 & 23.2 & 18.8 & 50.6 & -23.1 & 69.6 & 1.24 & 0.52 \\
\hline & T2-T0 & 7.5 & 19.3 & 50.4 & -40.1 & 55.2 & 0.39 & 0.97 \\
\hline & $\mathrm{T} 2-\mathrm{T} 1$ & -15.7 & 19.5 & 49.4 & -64.0 & 32.6 & -0.80 & 0.81 \\
\hline \multirow[t]{3}{*}{ Mean time high MET } & T1-T0 & 15.6 & 7.6 & 54.8 & -3.2 & 34.4 & 2.04 & 0.13 \\
\hline & T2-T0 & 15.7 & 7.9 & 55.0 & -3.7 & 35.1 & 2.00 & 0.14 \\
\hline & $\mathrm{T} 2-\mathrm{T} 1$ & 0.1 & 8.0 & 54.1 & -19.7 & 19.9 & 0.02 & 1.00 \\
\hline \multirow[t]{3}{*}{ 1STS } & T1-T0 & 0.6 & 1.9 & 66.0 & -4.0 & 5.3 & 0.34 & 0.98 \\
\hline & T2-T0 & 0.3 & 2.0 & 66.2 & -4.6 & 5.1 & 0.13 & 0.99 \\
\hline & $\mathrm{T} 2-\mathrm{T} 1$ & -0.4 & 20.1 & 65.7 & -5.31 & 4.5 & -0.20 & 0.99 \\
\hline \multirow[t]{3}{*}{ 6MWD } & T1-T0 & 1.2 & 17.9 & 68.9 & -42.5 & 44.9 & 0.07 & 0.99 \\
\hline & T2-T0 & -9.7 & 18.8 & 69.3 & -55.7 & 36.4 & -0.51 & 0.94 \\
\hline & $\mathrm{T} 2-\mathrm{T} 1$ & -10.9 & 18.9 & 68.6 & -57.1 & 35.4 & -0.58 & 0.91 \\
\hline
\end{tabular}

MET, metabolic equivalent of task; 1STS, one-minute sit to stand; 6MWD, six-minute walking distance; $\mathrm{SE}$, standard error; df, degrees of freedom; $\mathrm{Cl}$, confidence interval. Adjustment for multiple testing with sidak method

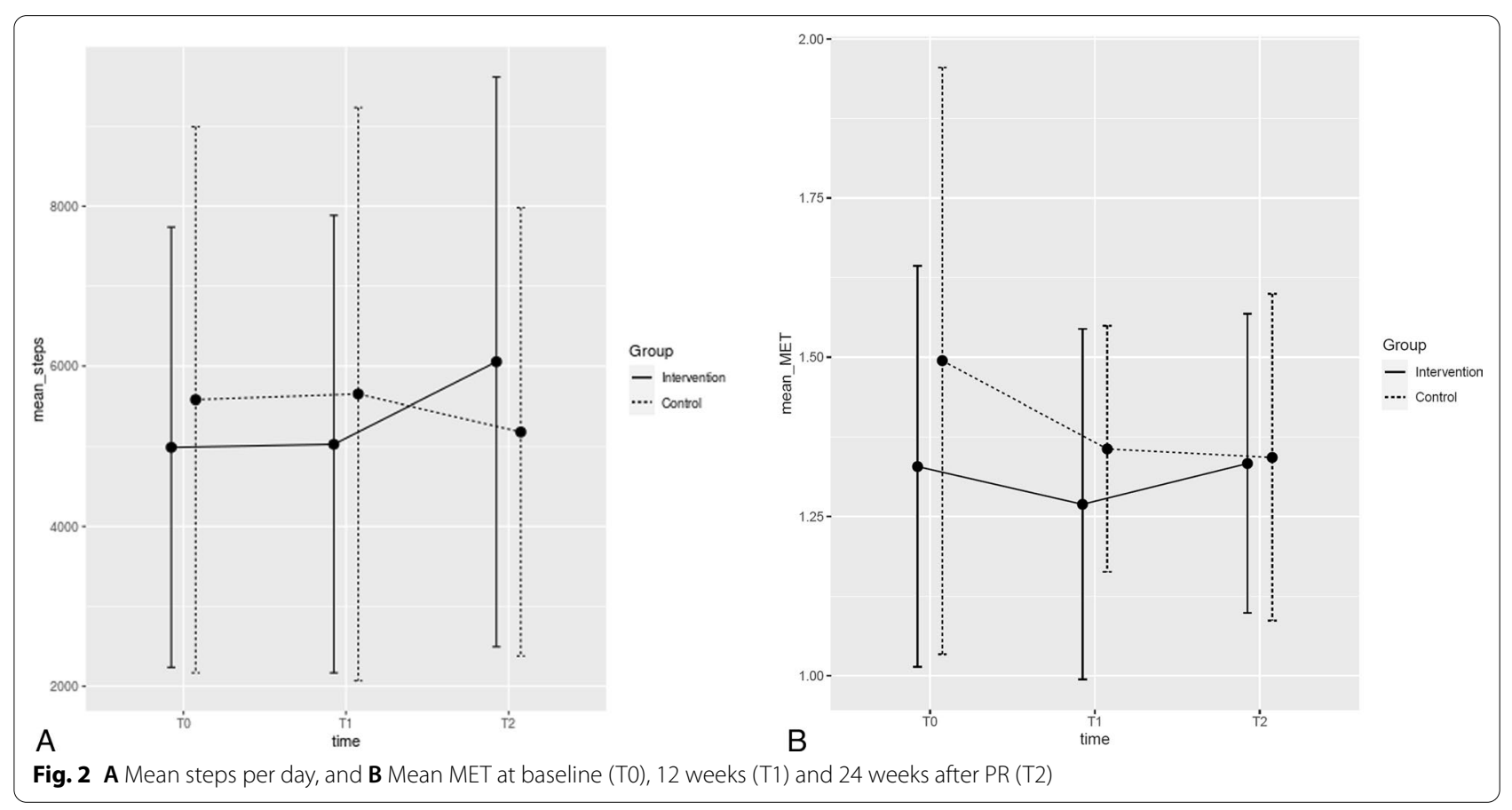


Table 5 PA stage of change assessment tool

\begin{tabular}{lllll}
\hline Stage of change & Group & T0 & T1 & T2 \\
\hline Pre-contemplation & Intervention & 0 & 0 & 0 \\
& Control & $1(6)$ & 0 & $1(8)$ \\
Contemplation & Intervention & $1(8)$ & 0 & $1(11)$ \\
& Control & $3(18)$ & 0 & 0 \\
Preparation & Intervention & $5(39)$ & 0 & 0 \\
Action & Control & $1(6)$ & $2(15)$ & 0 \\
\multirow{2}{*}{ Maintenance } & Intervention & $3(23)$ & $3(33)$ & 0 \\
& Control & $1(6)$ & $4(31)$ & $1(8)$ \\
& Intervention & $4(31)$ & $8(73)$ & $8(89)$ \\
& Control & $11(65)$ & $7(54)$ & $10(83)$
\end{tabular}

$\mathrm{n}$ (\%); Control group: T0 $\mathrm{n}=17,8$ missing; T1, $\mathrm{n}=13,12$ missing; $\mathrm{T} 2 \mathrm{n}=12,13$ missing; Intervention group: T0 $n=13,4$ missing; $T 1 \mathrm{n}=11,6$ missing; T2 $\mathrm{n}=9$, 8 missing

learning and use of MI as a communication technique in the context of behavioral change.

This study has some limitations. The main problem was the extremely slow inclusion rate of eligible patients. This situation persisted despite continual improvement in the screening procedures. Finally, the lockdown due to the Covid-19 pandemic caused the premature discontinuation of recruitment. This is the reason that the study is underpowered and that the findings should be interpreted with caution. During the long period of inclusion, some changes in clinical procedures were unavoidable due to routine quality improvements (e.g., exercise intervention was changed from large groups to small groups for better tailoring of individual exercise training) and staff turnover (the team responsible for exercise interventions changed). These quality improvement processes, and the individualization of PR may have caused a dilution of the counselling effects.

According to the original study protocol [31], the patients were to have been randomly allocated into blocks of four in each of two treatment arms. However, it became clear during the study that the randomization could not be implemented as planned. This limitation resulted in a different number of participants in the two arms of the study.

The accelerometer, SenseWear, is especially accurate and validated for this patient group [74, 75], but it not easily accessible to clinical practice. The device is more uncomfortable and less attractive than other more recently developed devices, such as a smart watch. Production of the SenseWear device was ceased in 2015, resulting in no technical support being available during the study. Some patients reported disturbing vibrations or sounds from the SenseWear during resting periods. Furthermore, the data quality was sometimes insufficient and had to be excluded from the analyses ( $\mathrm{n}=25$ data sets).

This study assessed PA stage of change in patients with COPD for the first time, to our knowledge. The resulting high number of missing questionnaires/nonresponses raises several questions. The PA stage of change assessment tool was translated into German using sound methodology; however, it was not validated in depth for patients with COPD. The low response rate could be due to a lack of understanding of the questionnaire, or it could be due to poor adherence. The general high proportion of missing patient reported outcomes in this study points towards the latter reason.

Although these limitations have had significant consequences for the study results, lessons have been learned on how to conduct a study in this setting. The lack of statistically significant findings does not necessarily mean that these effects do not exist. Future research with an adequate sample size, additional feedback strategy (e.g., pedometer), tailoring the MI intervention to people willing to change, might present a different picture of PA counselling added to a comprehensive PR.

\section{Conclusion}

Counselling, based on MI principles, which was embedded in a comprehensive PR program for people with COPD, did not show any significant effect on PA behavior, either during or after PR. The mode, timing and tailoring of the intervention, as well as sample size, need to be further investigated to determine the use of this potentially effective, patient-centered intervention in an outpatient PR setting.

\section{Acknowledgements}

The authors recognize the conceptual work of Prof Dr Arnoldus van Gestel, who regretfully passed away in June 2016. A special thank you to Christa Wachter, who was the contact person for participants and responsible for patient tracking and administration. Many thanks to Ilinka Tanic and Bianca Zehnder from Lungenfunktion KSW. Further thanks go to Sibylle Walder and Nicola Greco, the physiotherapists responsible for both exercise intervention and assessment, for their crucial contributions to this project. Last, but not least, we wish to thank all patients included in this project for their trust and participation.

\section{Authors' contributions}

MW, TH and AR were responsible for the conception of the project. SB was responsible for screening. AR and $A S$ were responsible for the counselling. SB, $S R$ and DG were responsible for the PR intervention. AM designed the data management concept. NS was the expert in Sensewear data management. DG, SB, SR and TH described the usual care procedure and PR. All authors contributed significantly to the interpretation of results. AR drafted the manuscript. All authors provided comments on each draft and approved the final version.

\section{Funding}

This study was funded by the Swiss Lung Association. Funding included the cost of devices (accelerometer) and some working hours. 


\section{Availability of data and materials}

The datasets analysed during the current study are ravailable from the corresponding author on reasonable request.

\section{Declarations}

\section{Ethics approval and consent to participate}

The regional ethics committee (EC,Canton Zurich) approved the study on $4^{\text {th }}$ May 2015 (PB_2016-01523). Data collection was conducted in accordance with Good Clinical Practice protocols and the Declaration of Helsinki principles. The COPD participants received written and oral information about the study. Written informed consent was obtained from all participants prior to baseline measurement.

\section{Consent for publication}

Not applicable.

\section{Competing interests}

All authors declare that they have no competing interests.

\section{Author details}

${ }^{1}$ Institute of Physiotherapy, School of Health Professions, ZHAW Zurich University of Applied Sciences, Katharina-Sulzer-Platz 9, 8400 Winterthur, Switzerland. ${ }^{2}$ Institute of Physiotherapy, Cantonal Hospital Winterthur, Brauerstrasse 15, 8401 Winterthur, Switzerland. ${ }^{3}$ Pneumology, Cantonal Hospital Winterthur, Brauerstrasse 15, 8401 Winterthur, Switzerland. ${ }^{4}$ Department of Pulmonology, University Hospital of Zurich, Raemistrasse 100, 8091 Zurich, Switzerland.

Received: 8 March 2021 Accepted: 23 September 2021

Published online: 12 October 2021

\section{References}

1. Nici L, Donner C, Wouters E, Zuwallack R, Ambrosino N, Bourbeau J, Carone M, Celli B, Engelen M, Fahy B, et al. American Thoracic Society/ European Respiratory Society statement on pulmonary rehabilitation. Am J Respir Crit Care Med. 2006;173(12):1390-413.

2. Garcia-Aymerich J, Farrero E, Felez MA, Izquierdo J, Marrades RM, Anto JM. Estudi del Factors de Risc d'Aguditzacio de la Mi: risk factors of readmission to hospital for a COPD exacerbation: a prospective study. Thorax. 2003;58(2):100-5.

3. Garcia-Aymerich J, Lange P, Benet M, Schnohr P, Anto JM. Regular physical activity reduces hospital admission and mortality in chronic obstructive pulmonary disease: a population based cohort study. Thorax. 2006;61(9):772-8.

4. Pitta F, Troosters T, Probst VS, Spruit MA, Decramer M, Gosselink R. Physical activity and hospitalization for exacerbation of COPD. Chest. 2006;129(3):536-44.

5. Waschki B, Kirsten A, Holz O, Muller KC, Meyer T, Watz H, Magnussen $\mathrm{H}$. Physical activity is the strongest predictor of all-cause mortality in patients with COPD: a prospective cohort study. Chest. 2011;140(2):331-42.

6. Vestbo J, Hurd SS, Agusti AG, Jones PW, Vogelmeier C, Anzueto A, Barnes PJ, Fabbri LM, Martinez FJ, Nishimura M, et al. Global strategy for the diagnosis, management, and prevention of chronic obstructive pulmonary disease: GOLD executive summary. Am J Respir Crit Care Med. 2013;187(4):347-65.

7. Donaire-Gonzalez D, Gimeno-Santos E, Balcells E, de Batlle J, Ramon MA, Rodriguez E, Farrero E, Benet M, Guerra S, Sauleda J, et al. Benefits of physical activity on COPD hospitalisation depend on intensity. Eur Respir J. 2015;46(5):1281-9.

8. Ward TJC, Plumptre CD, Dolmage TE, Jones AV, Trethewey R, Divall $P$, Singh SJ, Lindley MR, Steiner MC, Evans RA. Change in (V)over $\operatorname{dotO}(2$ peak) in response to aerobic exercise training and the relationship with exercise prescription in people with COPD a systematic review and meta-analysis. Chest. 2020;158(1):131-44.

9. Geidl W, Schlesinger S, Mino E, Miranda L, Pfeifer K. Dose-response relationship between physical activity and mortality in adults with noncommunicable diseases: a systematic review and meta-analysis of prospective observational studies. Int J Behav Nutr Phys Act. 2020;17(1):109.

10. Lopez-Campos JL, Soler-Cataluna JJ, Miravitlles M. Global strategy for the diagnosis, management, and prevention of chronic obstructive lung disease 2019 report: future challenges. Arch Bronconeumol. 2020;56(2):65-7.

11. Pulmonary rehabilitation. https://www.erswhitebook.org/chapters/ pulmonary-rehabilitation/.

12. Lima F, Altero G, Franco MC, Pinto R, Freire AP, Gomes P, Camillo CA Ramos D, Ramos EM. Factors influencing participation and adherence to a pulmonary rehabilitation program in patients with COPD: a qualitative study. Eur Respir J. 2016;48.

13. Guo SE, Bruce A. Improving understanding of and adherence to pulmonary rehabilitation in patients with COPD: a qualitative inquiry of patient and health professional perspectives. PLOS ONE. 2014;9:10.

14. Hayton C, Clark A, Olive S, Browne P, Galey P, Knights E, Staunton L, Jones A, Coombes E, Wilson AM. Barriers to pulmonary rehabilitation: characteristics that predict patient attendance and adherence. Respir Med. 2013;107(3):401-7.

15. Pitta F, Burtin C. Erratum to: The physical activity coach in pulmonary rehabilitation. In: Clini EM, Holland AE, Pitta F, Troosters T, editors. Textbook of pulmonary rehabilitation. Cham: Springer; 2017.

16. (UK) NGUT: NICE Guideline, No. 115. In: Self-management interventions, education and telehealth monitoring: chronic obstructive pulmonary disease in over 16s: diagnosis and management: evidence review C. edn. London: National Institute for Health and Care Excellence (UK); 2018.

17. Miller WR, Rollnick S. Motivational interviewing. Preparing people to change. 3rd ed. New London: The Guildford Press; 2013.

18. Zidarn M, Kolenko A. Effectiveness of motivational interview for smoking cessation in hospital setting. Eur Respir J. 2016;48.

19. Efraimsson EO, Fossum B, Ehrenberg A, Larsson K, Klang B. Use of motivational interviewing in smoking cessation at nurse-led chronic obstructive pulmonary disease clinics. J Adv Nurs. 2012;68(4):767-82.

20. Naderloo H, Vafadar Z, Eslaminejad A, Ebadi A. Effects of Motivational Interviewing on treatment adherence among patients with chronic obstructive pulmonary disease: a randomized controlled clinical trial. Tanaffos. 2018;17(4):241-9.

21. de Blok BM, de Greef MH, ten Hacken NH, Sprenger SR, Postema K, Wempe JB. The effects of a lifestyle physical activity counseling program with feedback of a pedometer during pulmonary rehabilitation in patients with COPD: a pilot study. Patient Educ Couns. 2006;61(1):48-55.

22. Burtin C, Langer D, van Remoortel $H$, Demeyer $H$, Gosselink $R$, Decramer M, Dobbels F, Janssens W, Troosters T. Physical activity counselling during pulmonary rehabilitation in patients with COPD: a randomised controlled trial. PLOS ONE. 2015;10(12):4989.

23. Altenburg WA, ten Hacken $\mathrm{NH}$, Bossenbroek $\mathrm{L}$, Kerstjens $\mathrm{HA}$, de Greef $M H$, Wempe JB. Short- and long-term effects of a physical activity counselling programme in COPD: a randomized controlled trial. Respir Med. 2015;109(1):112-21.

24. Cruz J, Brooks D, Marques A. Walk2Bactive: a randomised controlled trial of a physical activity-focused behavioural intervention beyond pulmonary rehabilitation in chronic obstructive pulmonary disease. Chron Respir Dis. 2016;13(1):57-66.

25. Lahham A, McDonald CF, Holland AE. Exercise training alone or with the addition of activity counseling improves physical activity levels in COPD: a systematic review and meta-analysis of randomized controlled trials. Int J Chron Obstruct Pulmon Dis. 2016;11:3121-36.

26. Benzo R, Vickers K, Novotny PJ, Tucker S, Hoult J, Neuenfeldt P, Connett J, Lorig K, McEvoy C. Health coaching and chronic obstructive pulmonary disease rehospitalization a randomized study. Am J Respir Crit Care Med. 2016;194(6):672-80.

27. Arbillaga-Etxarri A, Gimeno-Santos E, Barberan-Garcia A, Balcells E, Benet M, Borrell E, Celorrio N, Delgado A, Jane C, Marin A, et al. Long-term efficacy and effectiveness of a behavioural and community-based exercise intervention (Urban Training) to increase physical activity in patients with COPD: a randomised controlled trial. Eur Respir J. 2018;52:4.

28. Benzo RP, Kirsch JL, Hathaway JC, McEvoy CE, Vickers KS. Health coaching in severe COPD after a hospitalization: a qualitative analysis of a large randomized study. Respir Care. 2017;62(11):1403-11.

29. Prochaska JO, Velicer WF. The transtheoretical model of health behavior change. Am J Health Promot. 1997;12(1):38-48. 
30. Langer $\mathrm{D}$, Demeyer $\mathrm{H}$. Interventions to modify physical activity in patients with COPD: where do we go from here? Eur Respir J. 2016;48(1):14-7.

31. Rausch-Osthoff AK, Greco N, Schwank A, Beyer S, Gisi D, Scheermesser M, Meichtry A, Sievi N, Hess T, Wirz M. Effect of counselling during pulmonary rehabilitation on self-determined motivation towards physical activity in people with chronic obstructive pulmonary disease-protocol of a mixed methods study. BMC Pulm Med. 2017;17(1):115.

32. Spruit MA, Singh SJ, Garvey C, ZuWallack R, Nici L, Rochester C, Hill K, Holland AE, Lareau SC, Man WD, et al. An official American Thoracic Society/ European Respiratory Society statement: key concepts and advances in pulmonary rehabilitation. Am J Respir Crit Care Med. 2013;188(8):e13-64.

33. Maltais F, LeBlanc P, Jobin J, Berube C, Bruneau J, Carrier L, Breton MJ, Falardeau $G$, Belleau R. Intensity of training and physiologic adaptation in patients with chronic obstructive pulmonary disease. Am J Respir Crit Care Med. 1997;155(2):555-61

34. Bekkering EC, Hendriks HJM, Paterson WJ. Guidelines for physiotherapeutic management in chronic obstructive pulmonary disease (COPD). Phys Ther Rev. 2000;5:559-74.

35. Puhan MA, Schunemann HJ, Frey M, Scharplatz M, Bachmann LM. How should COPD patients exercise during respiratory rehabilitation? Comparison of exercise modalities and intensities to treat skeletal muscle dysfunction. Thorax. 2005;60(5):367-75.

36. Gloeckl R, Marinov B, Pitta F. Practical recommendations for exercise training in patients with COPD. Eur Respir Rev Off J Eur Respir Soc 2013;22(128):178-86.

37. Moyers TB, Rowell LN, Manuel JK, Ernst D, Houck JM. The Motivational Interviewing treatment integrity code (MITI 4): Rationale, preliminary reliability and validity. J Subst Abuse Treat. 2016;65:36-42

38. Moyers TB, Manuel JK, Ernst D. Die Deutsche Version des motivational interviewing treatment integrity coding manual 4.2.1 (MITI 4.2.1) Unpublished manual, deutsche Version. In. https://casaa.unm.edu/download/ miti_german.pdf; 2014.

39. Hettema JE, Ernst D, Williams JR, Miller KJ. Parallel processes: using motivational interviewing as an implementation coaching strategy. J Behav Health Serv Res. 2014;41(3):324-36.

40. Aliverti A, Macklem PT. The major limitation to exercise performance in COPD is inadequate energy supply to the respiratory and locomotor muscles. J Appl Physiol. 2008;105(2):749-51.

41. Craig CL, Marshall AL, Sjostrom M, Bauman AE, Booth ML, Ainsworth BE, Pratt M, Ekelund U, Yngve A, Sallis JF, et al. International physical activity questionnaire: 12-country reliability and validity. Med Sci Sports Exerc. 2003;35(8):1381-95.

42. Kim Y, Park I, Kang M. Convergent validity of the international physical activity questionnaire (IPAQ): meta-analysis. Public Health Nutr. 2013;16(3):440-52.

43. Markland D, Tobin V. A modification to the behavioural regulation in exercise questionnaire to include an assessment of amotivation. J Sport Exerc Psychol. 2004;26(2):191-6.

44. Wilson PM, Rodgers WM, Loitz CC, Scrime G. "It's who I am... really!"The importance of integrated regulation in exercise contexts. J Biobehav Res. 2006:11:79-104.

45. Prochaska JO, DiClemente CC. Transtheoretical therapy: towards a more integrative model of change. Psychother Theory Res Pract. 1982;19(3):276-88.

46. Guyatt GH, Berman LB, Townsend M, Pugsley SO, Chambers LW. A measure of quality of life for clinical trials in chronic lung disease. Thorax. 1987;42(10):773-8.

47. Reda AA, Kotz D, Kocks JW, Wesseling G, van Schayck CP. Reliability and validity of the clinical COPD questionniare and chronic respiratory questionnaire. Respir Med. 2010;104(11):1675-82.

48. Jones PW, Harding G, Berry P, Wiklund I, Chen WH, Kline Leidy N. Development and first validation of the COPD assessment test. Eur Respir J. 2009;34(3):648-54.

49. Sievi NA, Franzen D, Kohler M, Clarenbach CF. Physical inactivity and arterial stiffness in COPD. Int J Chron Obstruct Pulmon Dis. 2015;10:1891-7.

50. Watz $\mathrm{H}$, Waschki $\mathrm{B}$, Meyer $\mathrm{T}$, Magnussen $\mathrm{H}$. Physical activity in patients with COPD. Eur Respir J. 2009;33(2):262-72.

51. Bates D, Machler M, Bolker BM, Walker SC. Fitting linear mixed-effects models using Ime4. J Stat Softw. 2015;67(1):1-48.
52. A Language and Environment for Statistical Computing.

53. Demeyer H, Burtin C, Hornikx M, Camillo CA, Van Remoortel H, Langer D, Janssens W, Troosters T. The minimal important difference in physical activity in patients with COPD. PLOS ONE. 2016;11(4):e0154587.

54. Scheermesser M, Reicherzer L, Beyer S, Gisi D, Rezek S, Hess T, Wirz M, Rausch Osthoff AK. The influence of pulmonary rehabilitation and counselling on perceptions of physical activity in individuals with COPD—a qualitative study. Int J Chron Obstruct Pulmon Dis. 2021;16:2337-50.

55. O'Halloran PD, Blackstock F, Shields N, Holland A, lles R, Kingsley M, Bernhardt J, Lannin N, Morris ME, Taylor NF. Motivational interviewing to increase physical activity in people with chronic health conditions: a systematic review and meta-analysis. Clin Rehabil. 2014;28(12):1159-71.

56. Chapter 6 Intervention Mapping Step 3 Program design. In: Planning health promotion programs. Fourth edn. Edited by Bartholomew Eldredge LK, Markham CM, Ruiter RAC, Fernandez ME, Kok G, Parcel GS. San Francisco, USA: Jossey-Bass; 2016.

57. Mantoani LC, Rubio N, McKinstry B, MacNee W, Rabinovich RA. Interventions to modify physical activity in patients with COPD: a systematic review. Eur Respir J. 2016;48(1):69-81.

58. Organisation WH. World Health Organization. Implementation tools: package of essential noncommunicable (PEN) disease interventions for primary health care in low-resource settings. In. Edited by Organization WH. Geneva; 2013.

59. WHO Guidelines on Physical Activity and Sedentary Behaviour

60. Cindy Ng LW, Mackney J, Jenkins S, Hill K. Does exercise training change physical activity in people with COPD? A systematic review and metaanalysis. Chron Respir Dis. 2012;9(1):17-26.

61. Pitta F, Troosters T, Probst VS, Langer D, Decramer M, Gosselink R. Are patients with COPD more active after pulmonary rehabilitation? Chest. 2008;134(2):273-80.

62. Wempe JB, Wijkstra PJ. The influence of rehabilitation on behaviour modification in COPD. Patient Educ Couns. 2004;52(3):237-41.

63. Polkey MI, Rabe KF. Chicken or egg: physical activity in COPD revisited. Eur Respir J. 2009;33(2):227-9.

64. Blondeel A, Demeyer $H$, Janssens W, Troosters T. The role of physical activity in the context of pulmonary rehabilitation. COPD. 2018;15(6):632-9.

65. Holland AE, Mahal A, Hill CJ, Lee AL, Burge AT, Moore R, Nicolson C, O'Halloran P, Cox NS, Lahham A, et al. Benefits and costs of home-based pulmonary rehabilitation in chronic obstructive pulmonary disease-a multi-centre randomised controlled equivalence trial. BMC Pulm Med. 2013;13:57

66. Cox NS, Dal Corso S, Hansen H, McDonald CF, Hill CJ, Zanaboni P, Alison JA, O'Halloran P, Macdonald H, Holland AE. Telerehabilitation for chronic respiratory disease. Cochrane Database System Rev. 2021;1:CD013040.

67. Cox NS, McDonald CF, Alison JA, Mahal A, Wootton R, Hill CJ, Bondarenko J, Macdonald H, O'Halloran P, Zanaboni P, et al. Telerehabilitation versus traditional centre-based pulmonary rehabilitation for people with chronic respiratory disease: protocol for a randomised controlled trial. BMC Pulm Med. 2018;18(1):71.

68. Hansen $\mathrm{H}$, Bieler T, Beyer N, Kallemose T, Wilcke JT, Ostergaard LM, Frost Andeassen H, Martinez G, Lavesen M, Frolich A, et al. Supervised pulmonary tele-rehabilitation versus pulmonary rehabilitation in severe COPD: a randomised multicentre trial. Thorax. 2020;75(5):413-21.

69. Damhus CS, Emme C, Hansen H. Barriers and enablers of COPD telerehabilitation - a frontline staff perspective. Int J Chron Obstruct Pulmon Dis. 2018;13:2473-82

70. Rosenbek Minet L, Hansen LW, Pedersen CD, Titlestad IL, Christensen JK, Kidholm K, Rayce K, Bowes A, Mollegard L. Early telemedicine training and counselling after hospitalization in patients with severe chronic obstructive pulmonary disease: a feasibility study. BMC Med Inform Decis Mak. 2015;15:3

71. Oates GR, Niranjan SJ, Ott C, Scarinci IC, Schumann C, Parekh T, Dransfield MT. Adherence to pulmonary rehabilitation in copd a qualitative exploration of patient perspectives on barriers and facilitators. J Cardiopulm Rehabil Prev. 2019;39(5):344-9.

72. Weiterbildung für Gesundheitsfachpersonen.

73. Rehabilitation P. Das Lehr- und Lernbuch für dsa Reha-Team der D-A-CH Arbeitsgesmeinschaft Pneumologische Rehabilitation. München: DustriVeralg Dr.Karl Feistle; 2020. 
74. Van Remoortel H, Raste Y, Louvaris Z, Giavedoni S, Burtin C, Langer D, Wilson F, Rabinovich R, Vogiatzis I, Hopkinson NS, et al. Validity of six activity monitors in chronic obstructive pulmonary disease: a comparison with indirect calorimetry. PLoS ONE. 2012;7(6):e39198.

75. Hill K, Dolmage TE, Woon L, Goldstein R, Brooks D. Measurement properties of the sensewear armband in adults with chronic obstructive pulmonary disease. Thorax. 2010;65(6):486-91.

\section{Publisher's Note}

Springer Nature remains neutral with regard to jurisdictional claims in published maps and institutional affiliations.
Ready to submit your research? Choose BMC and benefit from:

- fast, convenient online submission

- thorough peer review by experienced researchers in your field

- rapid publication on acceptance

- support for research data, including large and complex data types

- gold Open Access which fosters wider collaboration and increased citations

- maximum visibility for your research: over $100 \mathrm{M}$ website views per year

At BMC, research is always in progress.

Learn more biomedcentral.com/submissions 